\title{
Antimicrobial usage in pig production: Effects on Escherichia coli virulence profiles and antimicrobial resistance
}

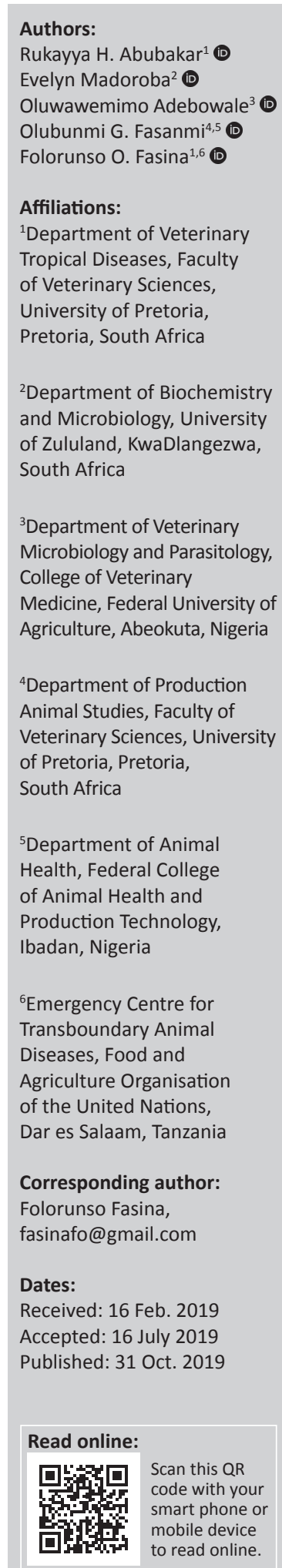

Antimicrobials (AM) are used for growth promotion and therapy in pig production. Its misuse has led to the development of resistant organisms. We evaluated Escherichia coli virulence genes, and compared phenotypic-genotypic antimicrobial resistance (AMR) patterns of faecal E. coli from pigs receiving routine farm treatment without antimicrobial agents against pigs treated routinely with AM over 70 days. Recovered E. coli were tested for AMR using disk diffusion and polymerase chain reaction. Virulence genes were detected in $24.8 \%$ of isolates from antimicrobial group and $43.5 \%$ from non-antimicrobial group $(p=0.002)$. The proportion of virulence genes heat-stable enterotoxins a \& b (STa, STb), enteroaggregative heat stable enterotoxin 1 [EAST1] and Shiga toxin type 2e [Stx2e]) were $18.1 \%, 0.0 \%, 78.7 \%$ and $3.0 \%$ for antimicrobial group and $14.8 \%, 8.5 \%, 85.1 \%$ and $12.7 \%$ for non-antimicrobial groups, respectively. Resistance to oxytetracycline was most common $(p=0.03)$ in samples collected between days 10 and 21. Resistance shifted to amoxicillin on days 56-70, and trimethoprim resistance was observed throughout. Seventeen phenotypic AMR combinations were observed and eight were multidrug resistant. At least one tetracycline resistance gene was found in $63.9 \%$ of the isolates. tet (A) $(23.3 \%)$ was most common in the antimicrobial group, whereas tet (B) $(43.5 \%)$ was prevalent in the nonantimicrobial group. Usage or non-usage of antimicrobial agents in growing pigs does not preclude virulence genes development and other complex factors may be involved as previously described. Heavily used AM correspond to the degree of resistance and tetracycline resistance genes were detected during the growth phase.

Keywords: antimicrobial; Escherichia coli; microbial drug resistance; virulence.

\section{Introduction}

Escherichia coli is a major cause of diarrhoea in pigs (piglets and weaners) at different levels of intensity worldwide (Vu Khac et al. 2006). In piglets, E. coli diarrhoea may be followed by terminal septicaemia, which is an important cause of economic loss for pig producers globally (Toledo et al. 2012). The estimated pig population in South Africa as of 2010 was about 1.5 million (Meissner, Scholtz \& Palmer 2013), while the population worldwide is about 1 billion. Pork serves as an important source of protein for human beings in developing countries or areas where pork consumption is not prohibited (Madzimure et al. 2012).

Diarrhoeagenic E. coli pathovars involved in pig enteric infections include mainly enterotoxigenic E. coli (ETEC) encoding heat-stable enterotoxins a \& b (STa, STb), enteroaggregative heat stable enterotoxin 1 [EAST1]) and/or heat-labile (LT) enterotoxins, causing secretory diarrhoea in newborn and weaned piglets (Gyles \& Fairbrother 2010). In addition, Shiga toxin E. coli (STEC) strains encode the Shiga toxin type 2e (Stx2e) that causes oedema disease but not diarrhoea (MacLeod, Gyles \& Wilcock 1991). Interestingly, some strains harbour both the Stx2e genes and enterotoxin genes capable of causing symptoms of both oedema disease and diarrhoea in the same animal (STEC/ETEC) (Barth, Schwanitz \& Bauerfeind 2011). Many porcine ETEC and STEC strains have fimbrial structures on their surface that like LT, STa and STb enterotoxins are usually plasmid mediated (Dubreuil, Isaacson \& Schifferli 2016). These fimbriae are termed colonisation antigens and they enable the bacteria to colonise the epithelial surface of the pig's small intestine, namely, F4 (K88), F5 (K99), F6 (P987), F18 and F41 usually found in pig ETEC (Blanco et al. 2006).

How to cite this article: Abubakar, R.H., Madoroba, E., Adebowale, O., Fasanmi, O.G. \& Fasina, F.O., 2019, 'Antimicrobial usage in pig production: Effects on Escherichia coli virulence profiles and antimicrobial resistance', Onderstepoort Journal of Veterinary Research 86(1), a1743. https://doi.org/10.4102/ojvr.v86i1.1743

Copyright: @ 2019 . The Authors. Licensee: AOSIS. This work is licensed under the Creative Commons Attribution License. 
Antimicrobial agents are frequently used in the treatment and control of these enteric infections in pigs.

A recent study has shown that administration of antimicrobial agents increases the risk of antimicrobial resistance (AMR) (Burow et al. 2014). Other factors like stress from temperature, crowding and management also seem to contribute to the occurrence of AMR in animals (Sørum \& Sunde 2001). The commensal bacteria in animals may become a reservoir of resistance to genes for pathogenic bacteria. This may contaminate meat and meat products meant for human consumption (Van den Bogaard \& Stobberingh 2000). Recent reports have indicated that the prevalence of antimicrobialresistant E. coli is on the increase (Luppi et al. 2015; Toledo et al. 2012) and the infections caused by the resistant bacteria usually fail to respond to treatment by specific antimicrobial agents (Rice 2009). This may be associated with the increased proliferation of bacterial pathogens, re-infection rates, chronicity, opportunistic infections with resistant organisms and a reduced life span (Capita \& Alonso-Calleja 2013).

Resistance to tetracycline determined phenotypically has been reported more frequently among bacteria isolated from pigs than previously known (Tadesse 2012). The resistance is known to be inducible and occurs basically because of the acquisition of tetracycline (tet) or oxytetracycline (otr) genes (Roberts 2011) and many isolates from pigs have shown multidrug resistance genes located on plasmids (Lutz et al. 2011).

Escherichia coli infections have been identified to be a challenge in the South African pig production industry (Fasina, Bwala \& Madoroba 2015; Kanengoni et al. 2017). A recent study showed that the prevalence of ETEC, STEC and EAST1 and associated fimbrial genes in indigenous South African breeds was high (Mohlatlole et al. 2013), an outbreak of multidrug resistance coliceptisaemia in weanling pigs was reported (Ikwap et al. 2016) and an investigation on piglet mortality in a farm was characterised to be associated with STEC (Kanengoni et al. 2017). Treatment and control of disease outbreaks in the South African pig industry involves the use of antimicrobial agents (Henton et al. 2011). The purpose of this investigation was to determine the effect of antimicrobial treatment on the prevalence of virulence genes and AMR in intestinal E. coli in growing pigs.

\section{Methodology \\ Approvals, animal care and welfare}

All pigs involved in the study were placed under a 24-hour monitoring programme conducted by the pig farm team (attendant and manager) for the duration of the study using the assessment and control of the severity of scientific procedures on laboratory animals scoring system and the guide to defining and implementing protocols for the welfare assessment of laboratory animals (Hawkins et al. 2011; Wallace et al. 1990). All piglets were housed in the farrowing unit with crates, creep area, heating lamps and unlimited access to the dam's teats, creep feed and water ad libitum (Figure 1). A total

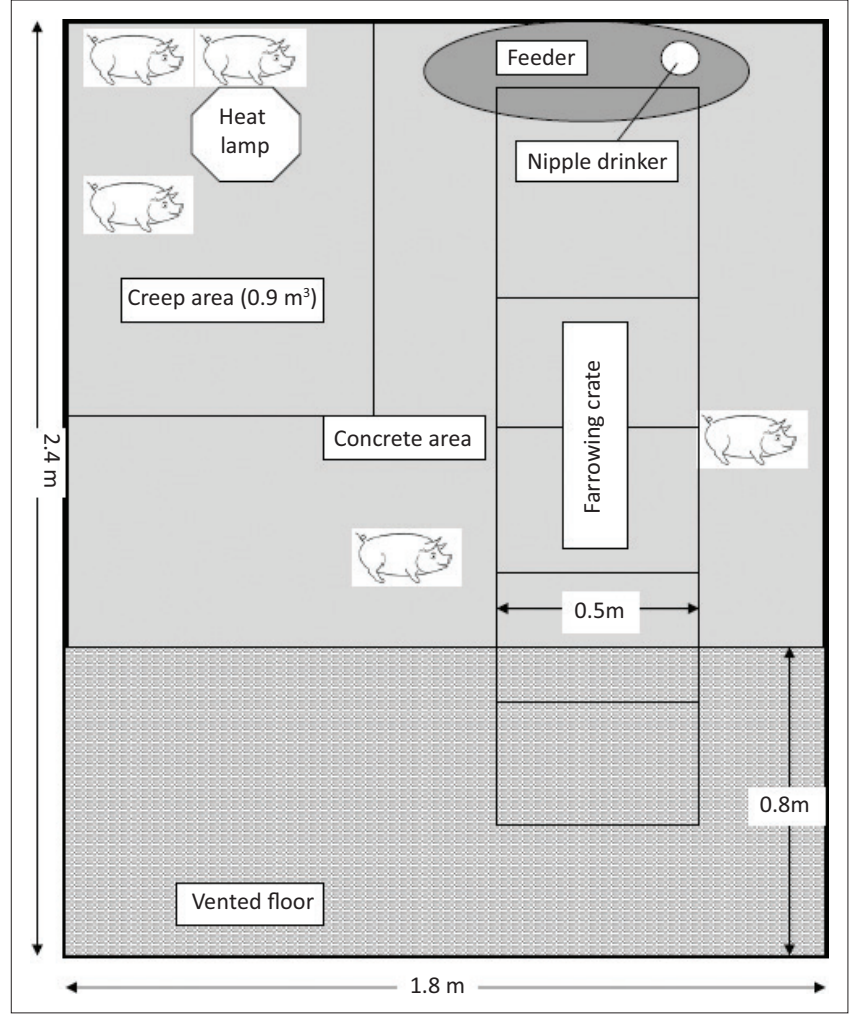

The sow (dam) is restricted to the farrowing crate for 4 weeks, but the piglets move freely within the pen. The creep area is warm with temperature range from $32^{\circ} \mathrm{C}$ (week 1) to $27^{\circ} \mathrm{C}$ (week 4). All pigs within a pen have access to feed and water liberally; however, the piglets depended primarily on sow's milk for the first 10-14 days. Only five piglets per litter were tagged for the experiment. After 4 weeks, the piglets are weaned into the weaners' pen. and vented floor.

of 4 out of 10 piglets were removed in the last 2 weeks of the study because of laboratory-confirmed colisepticaemia (oedema disease). For each animal to be removed by euthanasia (carbon dioxide asphyxiation in piglets or humane slaughter in weaners or growers) or sudden death, the humane endpoint was set with a Severity Index (SI) score of $>20$ on the Laboratory Animal Science Association (LASA) Working Party Scale (Wallace et al. 1990) and/or a score of $\geq 6$ on the BVAAWF/FRAME/RSPCA/UFAW Joint Working Group Scale (Hawkins et al. 2011). No other unexpected occurrence was recorded in the course of the experiment. The situation that triggered the scores for removal includes pain incompatible with animal welfare like prostration, nervous manifestation that affected normal movement and loss of the ability to ingest food for $24-48$ hours.

\section{Study design}

A small-scale commercial pig farm was identified in the Gauteng province of South Africa, and two pregnant sows were monitored clinically and physiologically until the day of farrowing. Piglets $(n=10)$ were randomly selected (five from each sow together with their unselected litter mates) and placed into two groups. All 10 selected piglets were tagged into groups A (non-antimicrobial group: with five tagged piglets and other non-tagged litter mates that were kept in one farrowing pen under routine farm management practices but without any form of antimicrobial usage) and B 
(antimicrobial group: with five piglets and other non-tagged litter mates that were kept under the routine management practices of the farm, which included administration of multivitamins, deworming, tail docking, vaccination, provision of warmth and antimicrobial administration to the sick animals). Effort was made to ensure the prevention of cross-contamination from the environment and between the groups by leaving three farrowing pens vacant ( $5.4 \mathrm{~m}$ width) between the two groups ensuring caretakers attend to the non-antimicrobial group before the antimicrobial group daily.

\section{Sample collection}

Rectal swabs were taken from all 10 piglets (four swabs per animal at each collection) with a sterile swab stick and each swab labelled with the specific pig identification number and age (days), and transported to the Agricultural Research Council-Onderstepoort Veterinary Research Feed and Food Analysis Laboratory (Bacteriology section) on ice. All samples were processed in the laboratory within $2 \mathrm{~h}$ of sample collection. The samples were collected periodically on days 1 , $5,10,21,28,35,56$ and 70 from all pigs.

\section{Classical microbiological analysis}

\section{Escherichia coli isolation and antimicrobial resistance testing}

The swabs were streaked directly on MacConkey agar (Oxoid, Basingstoke, United Kingdom [UK]) plates and incubated aerobically overnight at $37^{\circ} \mathrm{C}$. Lactose fermenting colonies $(n=4-6)$ were selected and sub-cultured on MacConkey agar. The pure colonies were then transferred to nutrient agar (Oxoid, Basingstoke, UK) plates. The isolates on nutrient agar plates were subjected to an indole test together with other biochemical reactions for E. coli identification. For this purpose, $10 \mathrm{~mL}$ of tryptone water was inoculated with pure culture and incubated over night at $37{ }^{\circ} \mathrm{C}$. Kovacs reagent (one to two drops) was added and the formation of a red ring was indicative of E. coli. In addition, an Indole test, Methyl red test, Voges-Proskauer test and a Citrate utilization test (IMViC) were also conducted. Subcultures were also cultured on 5\% sheep blood agar to determine the haemolytic characteristics of the E. coli. Escherichia coli ATCC 25922 and E. coli $\mathrm{O} 157$ were used as controls. Antimicrobial susceptibility testing was done using the Kirby-Bauer disk diffusion method. The zones were interpreted according to the Clinical Laboratory Standards Institute (CLSI) guidelines (Clinical Laboratory Standards Institute [CLSI] 2015).

The following antimicrobial agent discs were selected according to standard regulations (CLSI 2015; Food and Drug Administration 2012; World Health Organization 2016): amoxicillin (AML) $10 \mu \mathrm{g}$; cefotaxime (CTX) $30 \mu \mathrm{g}$; oxytetracycline (OT) $30 \mu \mathrm{g}$; kanamycin (K) $30 \mu \mathrm{g}$; florfenicol (FFC) $30 \mu \mathrm{g}$; enrofloxacin (ENR) $5 \mu \mathrm{g}$ and trimethoprim (W) $5 \mu \mathrm{g}$ (Table 1). The criteria that were used for selecting the antimicrobial agents are the use on the farm during pig production and the recommendations for testing of bacteria from animals (CLSI 2015). Escherichia coli isolates were considered to be multidrug resistant in cases of resistance to three or more classes of antimicrobial agents.

\section{Molecular characterisation of Escherichia coli isolates}

\section{DNA extraction and amplification using polymerase chain reaction}

DNA from E. coli isolates was obtained using the cell-lysis method by boiling at $95^{\circ} \mathrm{C}$ for 20 minutes to lyse the bacteria.

Escherichia coli isolates were tested for virulence genes and tetracycline resistance genes, tet (A, B, C and E) using sets of forward and reverse primers (Table 2). For detection of enterotoxin genes, STa, STb and heat-labile toxin (LT), a multiplex polymerase chain reaction (PCR) assay (Cheng et al. 2005) was adapted using a total $25 \mu \mathrm{L}$ of reaction volume including the PCR master mix (DreamTaqTM Green PCR Master Mix), $0.3 \mu \mathrm{L}$ of each primer, nuclease free PCR water (Fermentas) and $3 \mu \mathrm{L}$ deoxyribonucleic acid (DNA). Deoxyribonucleic acid amplification was carried out using Eppendorf Thermocycler (Eppendorf, Hamburg, Germany) and the cycling conditions were initial denaturation at $94{ }^{\circ} \mathrm{C}$ for $3 \mathrm{~min}$, followed by 10 cycles of denaturation at $94{ }^{\circ} \mathrm{C}$ for $30 \mathrm{~s}$, annealing at $60{ }^{\circ} \mathrm{C}-56^{\circ} \mathrm{C}\left(1{ }^{\circ} \mathrm{C}\right.$ decrease for every two cycles) for $30 \mathrm{~s}$ and extension at $72{ }^{\circ} \mathrm{C}$ for $1 \mathrm{~min}$, followed by another 22 cycles of similar thermocycling conditions but annealing at $56^{\circ} \mathrm{C}$, and a final extension at $72{ }^{\circ} \mathrm{C}$ for $10 \mathrm{~min}$.

The enteroaggregative heat stable enterotoxin 1 gene was detected using a monoplex PCR assay as described by Ngeleka and colleagues (Ngeleka et al. 2003) with slight

TABLE 1: Disk contents of antimicrobial and resistance break points used for disk diffusion testing of the Escherichia coli isolates ( $n=241)$.

\begin{tabular}{|c|c|c|c|c|c|}
\hline Antimicrobial class (FDA 2012) & Antibacterial agent & Abbreviations & Disk content $(\mu \mathrm{g})$ & Resistance break point (mm) & WHO Classification (WHO 2012) \\
\hline Penicillins & Amoxicillin & AML & 10 & $\leq 13$ & Critically important \\
\hline Cephems & Cefotaxime & CTX & 30 & $\leq 22$ & Critically important \\
\hline Tetracyclines & Oxytetracycline & OT & 30 & $\leq 11$ & Highly important \\
\hline Aminoglycosides & Kanamycin & K & 30 & $\leq 13$ & Critically important \\
\hline Phenicols & Florfenicol & FFC & 30 & $\leq 14$ & Highly important \\
\hline Flouroquinolones & Enrofloxacin & ENR & 5 & $\leq 16$ & Critically important \\
\hline Folate pathway inhibitors & Trimethoprim & W & 5 & $\leq 10$ & Highly important \\
\hline
\end{tabular}

Source: World Health Organization, 2016, Critically important antimicrobials for human medicine, viewed 09 February 2017, from https://apps.who.int/iris/bitstream/handle/10665/255027/ 9789241512220-eng.pdf; jsessionid=8830D9408E73E7DA20B1D1E741121B40?sequence=1.

Note: Breakpoints were based on Clinical Laboratory Standards Institute guideline (CLSI 2015) and Performance Standards for Antimicrobial Disk and Dilution Susceptibility Test for Bacteria Isolated from animals.

OT, oxytetracycline; AML, amoxicillin; W, trimethoprim; CTX, cefotaxime; K, kanamycin; ENR, enrofloxacin; FFC, florfenicol; WHO, World Health Organization; FDA, United States' Food and Drug Administration. 
TABLE 2: Primer sequences and amplicon sizes used for polymerase chain reaction detection of virulence genes, virulence factors and tetracycline resistance genes.

\begin{tabular}{|c|c|c|c|c|}
\hline Target gene & Primer & Primer sequence $\left(5^{\prime}-3^{\prime}\right)$ & Amplicon size (bp) & References \\
\hline \multicolumn{5}{|c|}{ Primer sequences for virulence genes and factors } \\
\hline \multicolumn{5}{|l|}{ Toxins } \\
\hline \multirow[t]{2}{*}{ EAST1 (astA) } & EAST-1-F & TCG GAT GCC ATC AAC ACA GT & 125 & Ngeleka et al. (2003) \\
\hline & EAST-1-R & GTC GCG AGT GAC GGC TTT GTA G & - & - \\
\hline \multirow[t]{2}{*}{ STa (estl) } & STa-F & GGG TTG GCA ATT TTT ATT TCT GTA & 183 & Cheng et al. (2006) \\
\hline & STa-R & ATT ACA ACA AAG TTC ACA GCA GTA & - & - \\
\hline \multirow[t]{2}{*}{ STb (estII) } & STb-F & ATG TAA ATA CCT ACA ACG GGT GAT & 360 & Cheng et al. (2006) \\
\hline & STb-R & TAT TTG GGC GCC AAA GCA TGC TCC & - & - \\
\hline \multirow[t]{2}{*}{ LT $(e / t)$} & LT-F & TAG AGA CCG GTATTA CAG AAATCT GA & 282 & Cheng et al. (2006) \\
\hline & LT-R & TCA TCC CGA ATT CTG TTA TAT ATGTC & - & - \\
\hline \multirow[t]{2}{*}{$\operatorname{Stx}_{1}(s t x l)$} & Stx1-F & ATT CGC TGA ATG TCATTC GCT & 664 & Cheng et al. (2006) \\
\hline & Stx1-R & ACG CTT CCC AGA ATT GCA TTA & - & - \\
\hline \multirow[t]{2}{*}{$\operatorname{Stx}_{2}(s t x I I)$} & Stx2-F & GAA TGA AGA AGA TGT TTA TAG CGG & 281 & Cheng et al. (2006) \\
\hline & Stx2-R & GGT TAT GCC TCA GTC ATT ATT AA & - & - \\
\hline $\operatorname{Stx}_{2 \mathrm{e}}(\operatorname{st} \times 2 e)$ & Stx2e-R & TTT TAT GGA ACG TAG GTA TTA CC & - & - \\
\hline \multicolumn{5}{|l|}{ Fimbriae } \\
\hline \multirow[t]{2}{*}{ F4 (K88) (faeG) } & F4 (K88)-F & GAT GAA AAA GAC TCT GAT TGC A & 841 & Cheng et al. (2006) \\
\hline & $\mathrm{F} 4$ (K88)-R & GAT TGC TAC GTT CAG CGG AGC G & - & - \\
\hline \multirow[t]{2}{*}{ F5 (K99) (fanA) } & F5 (K99)-F & CTG AAA AAA ACA CTG CTA GCT ATT & 543 & Cheng et al. (2006) \\
\hline & F5 (K99)-R & CAT ATA AGT GAC TAA GAA GGA TGC & - & - \\
\hline \multirow[t]{2}{*}{ F6 (987P) (fasA) } & F6 (987P)-F & GTT ACT GCC AGT CTA TGC CAA GTG & 463 & Cheng et al. (2006) \\
\hline & F6 (987P)-R & TCG GTG TAC CTG CTG AAC GAA TAG & - & - \\
\hline \multirow[t]{2}{*}{ F41 (fim41a) } & F41-F & GAT GAA AAA GAC TCT GAT TGC A & 682 & Cheng et al. (2006) \\
\hline & $\mathrm{F} 41-\mathrm{R}$ & TCT GAG GTC ATC CCA ATT GTG G & - & - \\
\hline \multirow[t]{3}{*}{$\mathrm{F} 18(\mathrm{fed} A)$} & $\mathrm{F} 18-\mathrm{F} 1(\mathrm{~b})$ & ATG AAA AGA CTA GTG TTT ATT TCT T & 513 or 516 & Cheng et al. (2005) \\
\hline & $\mathrm{F} 18-\mathrm{F} 2$ (c) & CGT GAA CGG TAA AAC ACA GGG & 170 & - \\
\hline & F18-R & TTA CTT GTA AGT AAC CGC GTA AGC C & - & - \\
\hline \multicolumn{5}{|l|}{ Adhesins } \\
\hline \multirow[t]{2}{*}{$\mathrm{EAE}(e a e)$} & EAE-F & CAT TAT GGA ACG GCA GAG GT & 790 & Ngeleka et al. (2003) \\
\hline & EAE-R & ATC TTC TGC GTA CTG CGT TCA & - & - \\
\hline \multirow[t]{2}{*}{ PAA (paa) } & PAA-F & ATG AGG AAC ATA ATG GCA GG & 360 & Ngeleka et al. (2003) \\
\hline & PAA-R & TCT GGT CAG GTC GTC AAT AC & - & - \\
\hline \multicolumn{5}{|c|}{ Primer sequences for tetracycline resistance genes } \\
\hline \multirow[t]{2}{*}{ tet $(\mathrm{A})(\operatorname{tet} A)$} & tetA-F & GCT ACA TCC TGC TTG CCT TC & 210 & Agga et al. (2014) \\
\hline & tetA-R & CAT AGA TCG CCG TGA AGA GG & - & - \\
\hline \multirow[t]{2}{*}{ tet (B) (tetB) } & tetB-F & TTG GTT AGG GGC AAG TTT TG & 659 & Agga et al. (2014) \\
\hline & tetB-R & GTA ATG GGC CAA TAA CAC CG & - & - \\
\hline \multirow[t]{2}{*}{ tet (C) (tetC) } & tetC-F & CTT GAG AGC CTT CAA CCC AG & 418 & Agga et al. (2014) \\
\hline & tetC-R & ATG GTC GTC ATC TAC CTG CC & - & - \\
\hline \multirow[t]{2}{*}{ tet (E) (tetE) } & tetE-F & AAA CCA CAT CCT CCA TAC GC & 278 & Agga et al. (2014) \\
\hline & tetE-R & AAA TAG GCC ACA ACC GTC AG & - & - \\
\hline
\end{tabular}

Source: Primer sequences were partially adapted from Mohlatlole, R.P., Madoroba, E., Muchadeyi, F.C., Chimonyo, M., Kanengoni, A.T. \& Dzomba, E.F., 2013, 'Virulence profiles of enterotoxigenic, Shiga toxin and enteroaggregative Escherichia coli in South African pigs', Tropical Animal Health and Production 45(6), 1399-1405. https://doi.org/10.1007/s11250-013-0377-4; Agga, G.E., Scott H.M., Amachawadi, R.G., Nagaraja, T.G., Vinasco, J., Bai J. et al., 2014, 'Effects of chlortetracycline and copper supplementation on antimicrobial resistance of fecal Escherichia coli from weaned pigs', Preventive Veterinary Medicine 114(3-4), 231-246. https://doi.org/10.1016/j.prevetmed.2014.02.010; Fasina, F.O., Bwala, D.G. \& Madoroba, E., 2015, 'Investigation of multidrug-resistant fatal colisepticaemia in weanling pigs', The Onderstepoort Journal of Veterinary Research 82(1), 986. https://doi.org/10.4102/ojvr.v82i1.986; Kanengoni, A.T., Thomas, R., Gelaw, A.K. \& Madoroba, E., 2017, 'Epidemiology and characterization of Escherichia coli outbreak on a pig farm in South Africa', FEMS Microbiology Letters 364(3). https://doi.org/10.1093/femsle/fnx010.

bp, base pairs; EAST1, enteroaggregative heat-stable 1; astA, enterotoxin 1; STa (estl), heat-stable enterotoxins; STb (estll), heat-stable enterotoxins; LT (elt), heat-labile enterotoxin; Stx (stxI), Shiga toxin; Stx ${ }_{2}\left(\right.$ stxll), Shiga toxin; $\operatorname{Stx}_{2 e}($ stx2e)), Shiga toxin; F4 (K88) (faeG), Fimbriae; F5 (K99) (fanA), Fimbriae; F6 (987P) (fasA), Fimbriae; F41 (fim41a), Fimbriae; F18 (fedA)), Fimbriae; AIDA-1 (aidA)), adhesin involved in diffuse adherence; EAE (eae), E. coli attaching and effacing; PAA (paa), porcine attaching and effacing-associated; tet (A) (tetA),Tetracycline; tet (B) (tetB), Tetracycline; tet adhesin involved in diffuse adherence; EAE (eae),
(C) (tetC), Tetracycline; tet (C) (tetE), Tetracycline.

modifications. The reaction mixture consisted of $0.3 \mu \mathrm{L}$ of each primer, $12.5 \mu \mathrm{L}$ of $2 \times$ PCR master mix (DreamTaqTM Green PCR Master Mix, Fermentas) and $3 \mu$ L DNA nuclease free PCR water (Fermentas) to make a $25 \mu \mathrm{L}$ reaction. Thermocycling conditions were initial denaturation at $94{ }^{\circ} \mathrm{C}$ for $3 \mathrm{~min}$, followed by 35 cycles of $94^{\circ} \mathrm{C}$ for $30 \mathrm{~s}$, annealing at $60{ }^{\circ} \mathrm{C}$ for $30 \mathrm{~s}$ and elongation at $72{ }^{\circ} \mathrm{C}$ for $30 \mathrm{~s}$, then a final elongation step at $72{ }^{\circ} \mathrm{C}$ for $5 \mathrm{~min}$. A multiplex assay for stx (including $\mathrm{stx}_{1}, \mathrm{stx}_{2}$ ) was carried out using similar reaction mixtures and thermocycling conditions for the protocol above except for the annealing temperature which was $58^{\circ} \mathrm{C}$. The adhesin involved in diffuse adherence (AIDA) 1 , E. coli attaching and effacing (eae) and porcine attaching and effacing-associated ( $p a a)$ were amplified as a multiplex PCR using similar protocols as that of EAST1. The fimbriae F4, F5, F6 and F41 (Fimbriae set 1) and F18ab and F18ac (Fimbriae set 2) (Cheng et al. 2005) were amplified using multiplex PCR. The reaction mixtures were similar to those 
of the enterotoxins; however, the thermocycling conditions were initial denaturation at $94^{\circ} \mathrm{C}$ for $3 \mathrm{~min}$, followed by first 10 cycles of denaturation at $94^{\circ} \mathrm{C}$ for $30 \mathrm{~s}$ annealing at $66^{\circ} \mathrm{C}$ $-62{ }^{\circ} \mathrm{C}\left(1{ }^{\circ} \mathrm{C}\right.$ decrease for every two cycles) for $30 \mathrm{~s}$, and elongation at $72{ }^{\circ} \mathrm{C}$ for $60 \mathrm{~s}$, then 22 cycles of similar conditions except for annealing at $62{ }^{\circ} \mathrm{C}$ for $30 \mathrm{~s}$ and a final elongation at $72{ }^{\circ} \mathrm{C}$ for $10 \mathrm{~min}$.

Tetracycline resistance genes tet (A, B, C and E) were detected using a multiplex PCR assay as described by Agga et al. (2014) with slight modifications. The $25 \mu \mathrm{L}$ reaction mixture consisted of $12.5 \mu \mathrm{L}$ of Dreamtaq mastermix, $0.3 \mu \mathrm{L}$ of each primer, $3 \mu \mathrm{L}$ crude DNA and $7.1 \mu \mathrm{L}$ of nuclease free PCR grade water (Table 2). The thermal cycling conditions consisted of an initial denaturation at $95{ }^{\circ} \mathrm{C}$ for $10 \mathrm{~min}$, followed by 30 cycles of denaturation at $94{ }^{\circ} \mathrm{C}$ for $30 \mathrm{~s}$, annealing at $60^{\circ} \mathrm{C}$ for $1.5 \mathrm{~min}$ (90 seconds) and elongation at $72{ }^{\circ} \mathrm{C}$ for $1.5 \mathrm{~min}$ (90 seconds), followed by a final elongation step at $72{ }^{\circ} \mathrm{C}$ for $10 \mathrm{~min}$. Positive controls were obtained from the Agricultural Research Council-Onderstepoort Veterinary Research (ARC-OVR) Bacteriology and Feed and Food laboratories culture collections. They include B41 (F5, F41 and STa), RCM39a (Stx , Stx $_{2}$ and eae), WL 187/16 (Stx2e), TPNB 137/16 (STa, STb and LT) and E. coli ATCC25922 (negative control).

\section{Statistical analysis}

All output data including the management and field parameters were entered into Microsoft Excel ${ }^{\circ}$ (Microsoft Inc., Redmond, Washington, United States). Data were filtered, harmonised and aligned with bacteriological results based on days of sample collections (positive and negative results) from antimicrobial and non-antimicrobial groups. Descriptive and analytical statistics were conducted using Minitab® 16. (Minitab Inc., State College, Pennsylvania, United States). Specifically, two by two tables were generated for results and the classical test of hypothesis was conducted using $\chi^{2}$ for all categorical variables. The $p$-value was set at an alpha of 0.05 as the cut-off for significance and $95 \%$ confidence interval (CI). Proportions were calculated with 95\% CI in Openepi ${ }^{\circledR}$ version 3.01 online calculator (Dean, Sullivan \& Soe 2015).

\section{Ethical considerations}

Prior to the commencement of the study, a completed study protocol was submitted to the National Department of Agriculture, Forestry and Fisheries, South Africa, for approval to carry out responsible infectious disease research with the approval reference number: $12 / 11 / 1 / 1 / 8$ of the Section 20 of the Animal Disease Act 35 of 1984, South Africa. This approval ensures the strict regulation and control of infectious pathogens, and minimises the risk of contamination of the environment and other pig farms. In addition, other necessary permits associated with the control of infectious materials were strictly adhered to including the 'permission to move animal products from the farm' and approval of the farm management. Secondly, the protocol on the adherence to animal welfare was submitted to the Animal Ethics Committee of the University of Pretoria and an approval number V029-16 was granted.

\section{Results}

Based on the evidence gathered from the farm, routine antimicrobial administrations included the intra-uterine suppository of OT within $6 \mathrm{~h}$ post-partum in sows, and the parenteral or intramuscular injection of antimicrobials (AM) (AML and penicillin-streptomycin combinations to diarrhoeic piglets during the growth phase). One millilitre of iron dextran and $2 \mathrm{~mL}$ of multivitamins were also injected on day 3 of birth to all piglets. The piglets were allowed unlimited access to milk from the dams' teats for the first 14 days after which creep starter feed was introduced to reduce suckling stress on the sows. Grower feed was introduced from about the fifth week of life of the weaned piglets and these feeds were changed to pig fatteners feed after the seventh week.

\section{Virulence genes and adhesion factors}

A total of $241 \mathrm{E}$. coli isolates were obtained from both groups, of which $55.2 \%(n=133)$ were from the antimicrobial group and $44.8 \%(n=108)$ were from the non-antimicrobial group $(p=0.02)$. From the 241 isolates, 33\% $(n=80)$ harboured virulence genes, $24.8 \%$ (95\% CI: $18.2-32.7)$ and $43.5 \%(95 \%$ CI: 34.5-52.9) coming from the antimicrobial $(n=33)$ and nonantimicrobial $(n=47)$ group isolates, respectively $(p=0.002)$.

Enteroaggregative heat stable enterotoxin 1 was the most prevalent virulence gene in both groups with $78.7 \%$ (95\% CI: $62.25-89.32)$ and $85.1 \%$ (95\% CI: 72.32-92.59) of the virulence genes observed in the antimicrobial and nonantimicrobial groups, respectively $(p=0.46)$ (Table 3$)$. The STa observed were $18.1 \%$ (95\% CI: $8.61-34.39$ ) and $14.8 \%$ (95\% CI: 7.40-27.68) in the antimicrobial and nonantimicrobial groups, respectively $(p=0.70)$. No STb was identified in the antimicrobial group and 8.5\% $(95 \%$ CI: 3.36-19.93) of the STbs were detected in the nonantimicrobial group $(p=0.09)$. The st $_{2 \mathrm{e}}$ gene was identified in both groups; $3 \%$ (95\% CI: 0.53-15.32) in the antimicrobial group and $12.7 \%(95 \% \mathrm{Cl}: 5.98-25.17)$ in the non-antimicrobial group ( $p=0.13$; Table 3$)$.

Of the adhesion factors for virulence genes, F6 (4.25\% [95\% CI: 1.17-14.25]) and EAE (eae) (2.10\% [95\% CI: 0.37-11.11]) were observed in the non-antimicrobial group with none in the antimicrobial group. However, the AIDA1 incidence was 3.0\% (95\% CI: $0.53-15.32)$ and $23.4 \%$ (95\% CI: 13.6-37.22) in the antimicrobial and non-antimicrobial groups, respectively $(p=$ 0.01); and for PAA (paa), the prevalence was $18.1 \%$ (95\% CI: 8.61-34.39) and $0 \%$ (95\%: 0.00-7.55) for the antimicrobial and non-antimicrobial groups, respectively $(p<0.005)$ (Table 3$)$.

We observed 12 pathotypes, with EAST1 being the most common in both groups: $60.6 \%$ (95\% CI: 43.64-75.32) and 61.7\% (95\% CI: $47.43-74.21)$ of the antimicrobial group and 
TABLE 3a: Frequency of Escherichia coli isolates, virulence genes, adhesion factors and pathotypes from Escherichia coli isolates with virulence genes.

\begin{tabular}{|c|c|c|c|c|c|}
\hline \multirow[t]{2}{*}{ Day } & \multicolumn{2}{|c|}{$\begin{array}{l}\text { Antimicrobial } \\
\text { group }(n=33)\end{array}$} & \multicolumn{2}{|c|}{$\begin{array}{l}\text { Non-antimicrobial } \\
\text { group }(n=47)\end{array}$} & \multirow[t]{2}{*}{$p$-value } \\
\hline & $n$ & $95 \%$ C.I & $n$ & $95 \%$ C.I & \\
\hline \multicolumn{6}{|c|}{ Frequency of virulence genes at sampling points } \\
\hline 0 & 6.0 & $1.67-19.61$ & 11.6 & $4.63-22.59$ & 0.480 \\
\hline 5 & 36.3 & $22.19-53.38$ & 17.0 & $8.88-30.14$ & 0.050 \\
\hline 10 & 18.1 & $8.61-34.39$ & 21.2 & $11.99-34.9$ & 0.730 \\
\hline 21 & 3.0 & $0.53-15.32$ & 0 & $0-7.5$ & 0.230 \\
\hline 28 & 0 & $0.0-10.43$ & 12.7 & $5.98-25.17$ & 0.030 \\
\hline 35 & 15.1 & $6.65-30.92$ & 53.1 & $39.23-66.67$ & 0.001 \\
\hline 56 & 6 & $1.67-19.61$ & 0 & $0-7.55$ & 0.090 \\
\hline 70 & 15.1 & $6.65-30.92$ & 6.3 & $2.19-17.16$ & 0.200 \\
\hline
\end{tabular}

Note: Significant $p$-values are presented in bold.

TABLE 3b: Frequency of Escherichia coli isolates, virulence genes, adhesion factors and pathotypes from Escherichia coli isolates with virulence genes.

\begin{tabular}{|c|c|c|c|c|c|}
\hline \multirow[t]{2}{*}{ tet genes } & \multicolumn{2}{|c|}{$\begin{array}{l}\text { Antimicrobial } \\
\text { group }(n=133)\end{array}$} & \multicolumn{2}{|c|}{$\begin{array}{l}\text { Non-antimicrobial } \\
\text { group }(n=108)\end{array}$} & \multirow[t]{2}{*}{$p$-value } \\
\hline & $n$ & $95 \%$ C.I & $n$ & $95 \%$ C.I & \\
\hline \multicolumn{6}{|c|}{ Virulence genes Genes } \\
\hline Sta & 18.1 & $8.61-34.39$ & 14.8 & $7.40-27.68$ & 0.7000 \\
\hline STb & 0 & $0.0-10.43$ & 8.5 & $3.36-19.93$ & 0.0900 \\
\hline EAST1 & 78.7 & $62.25-89.32$ & 85.1 & $72.32-92.59$ & 0.4600 \\
\hline StX2e & 3 & $0.53-15.32$ & 12.7 & $5.98-25.17$ & 0.1300 \\
\hline \multicolumn{6}{|c|}{ Adhesion factors from isolates that carried virulence genes } \\
\hline AIDA & 3.0 & $0.53-15.32$ & 23.4 & $13.6-37.22$ & 0.0100 \\
\hline PAA & 18.1 & $8.61-34.39$ & 0 & $0-7.55$ & $<0.0050$ \\
\hline EAE & 0 & $0.0-10.43$ & 2.1 & $0.37-11.11$ & 0.4000 \\
\hline F6 & 0 & $0.0-10.43$ & 4.25 & $1.17-14.25$ & 0.2300 \\
\hline \multicolumn{6}{|c|}{ Pathotype combinations of isolates that carried virulence genes } \\
\hline \multicolumn{6}{|l|}{ Pathotypes } \\
\hline EAST1 & 60.6 & $43.64-75.32$ & 61.7 & $47.43-74.21$ & 0.9200 \\
\hline Sta & 18.1 & $8.61-34.39$ & 0 & $0-7.55$ & $<0.0050$ \\
\hline STa/F6 & 0 & $0.0-10.43$ & 2.1 & $0.37-11.11$ & 0.4000 \\
\hline STb/EAST1/AIDA1 & 0 & $0.0-10.43$ & 8.5 & $3.36-19.93$ & 0.0900 \\
\hline $\operatorname{Stx}_{2 \mathrm{e}}$ & 3.0 & $0.53-15.32$ & 0 & $0-7.55$ & 0.2300 \\
\hline EAST1/EAE & 0 & $0.0-10.43$ & 2.1 & $0.37-11.11$ & 0.4000 \\
\hline EAST1/PAA & 15.1 & $6.65-30.92$ & 0 & $0-7.55$ & $<0.0100$ \\
\hline EAST1/AIDA1 & 3.0 & $0.53-15.32$ & 8.5 & $3.36-19.93$ & 0.3200 \\
\hline EAST1/Sta & 0 & $0.0-10.43$ & 2.1 & $0.37-11.11$ & 0.4000 \\
\hline EAST1/STa/F6 & 0 & $0.0-10.43$ & 2.1 & $0.37-11.11$ & 0.4000 \\
\hline STa/Stx2e/AIDA & 0 & $0.0-10.43$ & 6.3 & $2.19-17.16$ & 0.1400 \\
\hline $\mathrm{STa} / \mathrm{Stx} 2 \mathrm{e}$ & 0 & $0.0-10.43$ & 6.3 & $2.19-17.16$ & 0.1400 \\
\hline \multicolumn{6}{|c|}{ Frequency of each of the tet genes observed } \\
\hline tet $\mathrm{A}$ & 23.3 & $16.9-31.1$ & 18.5 & $12.3-26.8$ & 0.3700 \\
\hline tet B & 21.0 & $14.9-28.7$ & 43.5 & $34.5-52.9$ & $<0.0005$ \\
\hline tet $\mathrm{C}$ & 20.3 & $14.3-27.9$ & 9.2 & $5.1-16.2$ & 0.0200 \\
\hline tet $\mathrm{E}$ & 12.7 & $8.1-19.5$ & 1.8 & $0.5-6.5$ & 0.0020 \\
\hline
\end{tabular}

Note: Significant $p$-values are presented in bold.

STa, heat-stable enterotoxins a; STb, heat-stable enterotoxins b; EAST 1, enteroaggregative heat-stable 1; StX2e, enterotoxin 1-Shiga toxin; AIDA, adhesin involved in diffuse adherence; PAA, porcine attaching and effacing-associated; EAE, E. coli attaching and effacing. F6, fimbriae; tet, tetracycline.

non-antimicrobial groups, respectively $(p=0.92)$ (Table 3 ). The trend for the recovery of virulence genes is available in Figure 2 with significant differences in recovery rates between antimicrobial and non-antimicrobial groups on days 5, 28 and 35 .

\section{Antimicrobial resistance}

In total, $164(68 \%)$ of the isolates showed phenotypic resistance to the seven antimicrobial agents tested; there was a significant difference $(p=0.02)$ between the antimicrobial group (61.6\% [95\% CI: 53.17-69.48]) and the non-antimicrobial group $(75.9 \% \quad[95 \%$ CI: 67.06-83.01]). Resistance to oxytetracycline (OT) was most common in the antimicrobial group; it accounted for 59.3\% (95\% CI: 50.9-67.3). In the nonantimicrobial group, $73.1 \%$ (95\% CI: $64.1-80.6)$ of the isolates also showed resistance to OT (Figure 3). The difference between the two groups was statistically significant $(p<0.05)$. Trimethoprim was the second antimicrobial that showed a high resistance 20.3\% (95\% CI: 14.3-27.9), followed by AML 12.7\% (95\% CI: 8.1-19.5), K (kanamycin) 6.7\% (95\% CI: 3.6-12.3), CTX 1.5\% (95\% CI: 0.4-5.3) and no resistance was detected for ENR and FFC in the antimicrobial group. Amoxicillin was the second most resistant antimicrobial 32.4\% (95\% CI: 24.3-41.7), followed by W (trimethoprim) $29.6 \%$ (95\% CI: 21.8-38.8), CTX 13.8\% (95\% CI: 8.6-21.6), K $7.4 \%$ (95\% CI: 3.8-13.9), ENR 4.6\% (95\% CI: $1.9-10.3)$ and no resistance to FFC was observed within the non-antimicrobial group isolates (Figure 3).

During the experimental period (days 1-70), resistance to OT was exhibited more commonly on day $21(p=0.01)$, which constituted about $25.3 \%$ (95\% CI: $17.0-35.8)$ in the antimicrobial group. However, in the non-antimicrobial group, OT resistance was more frequent on day 10, constituting $22.7 \%$ (95\% CI: 14.9-33.1). Amoxicillin resistance was more common on day 10 , and constituted $23.5 \%$ (95\% CI: 9.5-47.2) in the antimicrobial group and on days 10 and 35 , resulting in $28.5 \%$ (95\% CI: $16.3-45.0)$ in the nonantimicrobial group. Trimethoprim resistance was more frequent on day 21 , and constituted $37.0 \%$ (95\% CI: $21.5-55.7)$ in the isolates of the antimicrobial group and on day 10, and $40.6 \%$ (95\% CI: $25.5-57.7$ ) in the non-antimicrobial group. Cefotaxime resistance was observed only on days 0 and 28 in the isolates of the antimicrobial group. Escherichia coli resistance to CTX was observed only on days 5, 10 and 21 in the non-antimicrobial group. Kanamycin resistance was observed only on days 21 and 56 among the antimicrobial group isolates and on days 5, 10 and 21 among the nonantimicrobial group isolates (Table 4).

A total of 17 phenotypic AMR combinations were observed. Oxytetracycline phenotype was most common in the two groups, with $54.8 \%$ (95\% CI: 44.1-65.1) in the antimicrobial group and $40.2 \%$ (95\% CI: 30.3-51.0) among the nonantimicrobial group isolates $(p=0.06)$. However, OT-W-K and OT-AML-W-CTX-K were statistically significant $(p>0.05)$ among all the phenotypes observed in the two groups (Figure 4a).

\section{Tetracycline resistance genes}

Only 154 (64\%) of the isolates possessed one or more of the four tetracycline resistance genes tested; it constituted $64.6 \%$ (95\% CI: 56.2-72.2) of the antimicrobial group isolate population and $62.9 \%$ (95\% CI: $53.5-71.4)$ of the nonantimicrobial group isolates population.

The most frequently observed tet gene in the antimicrobial group is the tet (A), which constituted $23.3 \%$ (95\% CI: $16.9-31.1)$ 


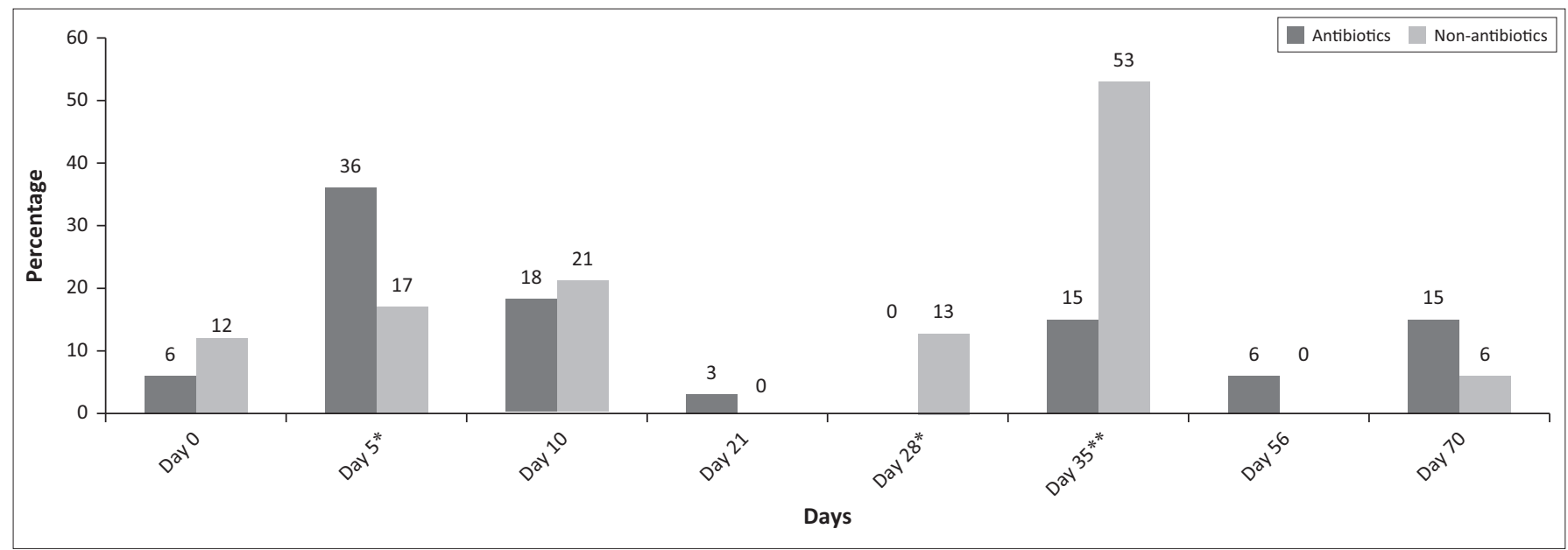

$*$, significant at $<0.05 ; * *$, significant at $<0.005$.

FIGURE 2: Frequency of isolation of virulence genes in percentages based on days of sampling.



OT, Oxytetracycline; AML, Amoxicillin; W, Trimethoprim; CTX, Cefotaxime; K, Kanamycin ENR, Enrofloxacin; FFC, Florfenicol.

$*$, significant value $<0.05 ; * *$, significant value $<0.005 ; * *$, significant value $<0.01$. FIGURE 3: Frequency of occurrence of antimicrobial resistance for each of the antimicrobial agents tested in this study.

of the isolates population, followed by tet (B) that made up $21.0 \%$ (95\% CI: 14.9-28.7), tet (C) (20.3\% [95\% CI: 14.3-27.9]) and tet (E) $(12.7 \% \quad[95 \% \quad \mathrm{CI}: 8.1-19.5])$. In the nonantimicrobial group isolates, tet (B) was more frequent (43.5\% [95\% CI: 34.5-52.9]), followed by tet (A) (18.5\% [95\% CI: $12.3-26.8])$, tet (C) $(9.2 \%$ [95\% CI: 5.1-16.2]) and tet (E) (1.8\% [95\% CI: 0.5-6.5]), with a significant statistical difference between the two groups (Table 3 ).

Eleven tet gene combinations were observed with the tet $(A)$ genotype being the most common in the antimicrobial group $33.7 \%$ (95\% CI: 24.6-44.2) and the tet (B) genotype 55.8\% (95\% CI: 44.0-67.0) among the non-antimicrobial group (Figure $4 \mathrm{~b}$ ).

\section{Discussion}

In this work, we compared the effect of usage and non-usage of $\mathrm{AM}$ in pig production from piglet to porker stage (1 to 70-110 days) using phenotypic and genotypic characteristics. Escherichia coli isolates were obtained in both groups of pigs (with or without AM) and differential resistance levels were observed in both groups. A total of 241 resident $E$. coli isolates were obtained from the samples, but no distinction was made between the commensal and pathogenic organisms in this study. A previous study had confirmed that animals with intense antimicrobial administration are more likely to present with AMR clinical isolates compared with the nonantimicrobial treated group that may have more commensal E. coli (Enne et al. 2008). Although a distance of over $5 \mathrm{~m}$ was created between the two experimental pens during this experiment with a view to circumvent cross-contamination, a resistance gene pattern was randomly observed in the two groups. Other workers have confirmed that mobile genetic elements allow for the horizontal gene transfer (HGT) of resistance genes to other pathogens, commensal and environmental strains (Muniesa, Colomer-Lluch \& Jofre 2013; Tripathi \&Tripathi 2017).

Virulence genes have been identified in both groups irrespective of whether AM were applied or not, with a higher prevalence in the non-AM group. It is highly likely that such genes are inclusive of the environmentally acquired horizontal gene transfer (HGT) of commensal E. coli. This observation is in agreement with the findings of other workers (Chapman et al. 2006), who stated that although commensal E. coli isolates are non-pathogenic, they may potentially contain virulence genes that are capable of causing disease (Chapman et al. 2006).

The enteroaggregative heat stable enterotoxin 1 was the predominant virulence gene, but no significant difference was detected between the antimicrobial and non-antimicrobial groups. Previous studies have concluded that EAST1 was a major determinant in E. coli-associated diarrhoea in pigs (Choi et al. 2001; Osek 2003). Although F4 that is associated with more severe diarrhoea, has been reported to be a more predominant fimbria found in pigs (Luppi et al. 2016). Our study identified only F6, which causes a milder form of diarrhoea in pigs. In addition, AIDA1 and PAA were the predominant adhesion factors detected in our study, but other factors were similarly recovered. AIDA1 has been associated with ETEC toxin genes (Sta, STb and EAST1) and Stx2e. The AIDA1 association with toxin genes has been identified in previous studies and indicated as an important marker gene 
TABLE 4: Frequency of occurrence of phenotypic antimicrobial resistance during the growing period.

\begin{tabular}{|c|c|c|c|c|c|c|}
\hline \multirow[t]{2}{*}{$\begin{array}{l}\text { Antimicrobial } \\
\text { agent }\end{array}$} & \multirow[t]{2}{*}{$\begin{array}{l}\text { Age } \\
\text { (days) }\end{array}$} & \multicolumn{2}{|c|}{$\begin{array}{l}\text { Antimicrobial } \\
\text { group } \dagger\end{array}$} & \multicolumn{2}{|c|}{$\begin{array}{c}\text { Non-antimicrobial } \\
\text { group }\end{array}$} & \multirow[t]{2}{*}{$p$-value } \\
\hline & & $n$ & $95 \%$ C.I & $n$ & $95 \%$ C.I & \\
\hline \multirow[t]{8}{*}{ Oxytetracycline } & 0 & 15.1 & $8.9-24.7$ & 13.9 & $7.9-23.2$ & 0.8200 \\
\hline & 5 & 7.5 & $3.5-15.5$ & 15.1 & $8.9-24.7$ & 0.1300 \\
\hline & 10 & 8.8 & $4.3-17.1$ & 22.7 & $14.9-33.1$ & $<0.0500$ \\
\hline & 21 & 25.3 & $17.0-35.8$ & 10.1 & $5.2-18.7$ & 0.0100 \\
\hline & 28 & 12.6 & $7.0-21.7$ & 15.1 & $8.9-24.7$ & 0.6500 \\
\hline & 35 & 16.4 & $9.8-26.1$ & 13.9 & $7.9-23.2$ & 0.6600 \\
\hline & 56 & 6.3 & 2.7-13.9 & 3.7 & $1.3-10.5$ & 0.4700 \\
\hline & 70 & 7.5 & $3.5-15.5$ & 5.0 & $1.9-12.3$ & 0.5100 \\
\hline \multirow[t]{8}{*}{ Amoxicillin } & 0 & 17.6 & $6.1-41.0$ & 2.8 & $0.5-14.5$ & 0.0600 \\
\hline & 5 & 5.8 & $1.0-26.9$ & 11.4 & $4.5-25.9$ & 0.5300 \\
\hline & 10 & 23.5 & $9.5-47.2$ & 28.5 & $16.3-45.0$ & 0.7000 \\
\hline & 21 & 11.7 & $3.2-34.3$ & 20 & $10.0-35.8$ & 0.4600 \\
\hline & 28 & 5.8 & $1.0-26.9$ & 8.5 & $2.9-22.3$ & 0.7300 \\
\hline & 35 & 5.8 & $1.0-26.9$ & 28.5 & $16.3-45.0$ & 0.0600 \\
\hline & 56 & 11.7 & $3.2-34.3$ & 0 & $0.0-9.8$ & $<0.0500$ \\
\hline & 70 & 17.6 & $6.1-41.0$ & 0 & $0.0-9.8$ & 0.0100 \\
\hline \multirow[t]{8}{*}{ Trimethoprim } & 0 & 11.1 & $3.8-28.0$ & 6.2 & $1.7-20.1$ & 0.5000 \\
\hline & 5 & 0 & $0.0-12.4$ & 15.6 & $6.8-31.7$ & $<0.0500$ \\
\hline & 10 & 0 & $0.0-12.4$ & 40.6 & $25.5-57.7$ & $<0.0005$ \\
\hline & 21 & 37.0 & $21.5-55.7$ & 3.1 & $0.5-15.7$ & 0.0010 \\
\hline & 28 & 7.4 & $2.0-23.3$ & 12.5 & $4.9-28.0$ & 0.5200 \\
\hline & 35 & 11.1 & $3.8-28.0$ & 21.8 & $11.0-38.7$ & 0.2700 \\
\hline & 56 & 18.5 & $8.1-36.7$ & 0 & $0.0-10.7$ & 0.0100 \\
\hline & 70 & 14.8 & $5.9-32.4$ & 0 & $0.0-10.7$ & $<0.0500$ \\
\hline \multirow[t]{8}{*}{ Cefotaxim } & 0 & 50 & $9.4-90.5$ & 0 & $0.0-20.3$ & 0.0050 \\
\hline & 5 & 0 & $0.0-65.7$ & 20 & $7.0-45.1$ & 0.4900 \\
\hline & 10 & 0 & $0.0-65.7$ & 73.3 & $48.0-89.1$ & $<0.0500$ \\
\hline & 21 & 0 & $0.0-65.7$ & 6.6 & $1.1-29.8$ & 0.7000 \\
\hline & 28 & 50 & $9.4-90.5$ & 0 & $0.0-20.3$ & 0.0050 \\
\hline & 35 & - & - & - & - & - \\
\hline & 56 & - & - & - & - & - \\
\hline & 70 & - & - & - & - & - \\
\hline \multirow[t]{8}{*}{ Kanamycin } & 0 & - & & - & - & - \\
\hline & 5 & 0 & $0.0-29.9$ & 12.5 & $0.1-49.2$ & 0.27 \\
\hline & 10 & 0 & $0.0-29.9$ & 75.0 & $40.1-93.7$ & 0.001 \\
\hline & 21 & 88.8 & $56.5-98.0$ & 12.5 & $0.1-49.2$ & $<0.005$ \\
\hline & 28 & - & & - & - & - \\
\hline & 35 & - & & - & - & - \\
\hline & 56 & 11 & $1.9-43.5$ & 0 & $0.0-32.4$ & 0.33 \\
\hline & 70 & - & - & - & - & - \\
\hline \multirow[t]{8}{*}{ Enrofloxacin } & 0 & 0 & - & 0 & - & - \\
\hline & 5 & 0 & - & 0 & - & - \\
\hline & 10 & 0 & - & 0 & - & - \\
\hline & 21 & 0 & - & 80.0 & $36.0-98.0$ & - \\
\hline & 28 & 0 & - & 20.0 & $2.0-64.0$ & - \\
\hline & 35 & 0 & - & 0 & - & - \\
\hline & 56 & 0 & - & 0 & - & - \\
\hline & 70 & 0 & - & 0 & - & - \\
\hline \multirow[t]{8}{*}{ Florfenicol } & 0 & 0 & - & 0 & - & - \\
\hline & 5 & 0 & - & 0 & - & - \\
\hline & 10 & 0 & - & 0 & - & - \\
\hline & 21 & 0 & - & 0 & - & - \\
\hline & 28 & 0 & - & 0 & - & - \\
\hline & 35 & 0 & - & 0 & - & - \\
\hline & 56 & 0 & - & 0 & - & - \\
\hline & 70 & 0 & - & 0 & - & - \\
\hline
\end{tabular}

Note: Significant values are presented in bold. All isolated bacteria were sensitive to florfenicol and enrofloxacin except for four and one isolates against enrofloxacin on days 21 and 28 in the non-antimicrobial group. Values in the brackets are $95 \%$ confidence intervals and bold $p$-values indicated significance difference between the groups.

$\dagger$, For the antimicrobial groups, the total numbers of samples that showed resistance were $79,17,27,2$ and 9 for oxytetracycline, amoxicillin, trimethoprim, cefotaxim and kanamycin respectively.

$\$$, For the non-antimicrobial groups, the total numbers of samples that showed resistance were $79,35,32,15,8,5$ and 0 for oxytetracycline, amoxicillin, trimethoprim, cefotaxim, kanamycin, enrofloxacin and florfenicol, respectively. for the causation of diarrhoea and oedema disease in pigs (Ngeleka et al. 2003; Zhao et al. 2009).

While a number of the combination of pathotypes were recovered in this study, no stx $x_{1}$ and $s t x_{2}$ was identified. These toxins have been more commonly isolated in bovine, ovine and in humans in cases of haemolytic ureamic syndrome (Paton \& Paton 1998). Furthermore, pigs have not been known as reservoirs for human pathogenic STEC (Hawkins et al. 2011). Stx $x_{2 e}$ isolation was higher in the non-antimicrobial group and was commonly associated with AIDA, perhaps because of a lack of maternal immunity and the development of oedema disease because no antimicrobial was used to clear the pathogenic organisms. Oedema disease affects pigs during the post-weaning period with high mortality and no recorded commercial vaccine is available, but reports have shown that high sero-prevalence for $\mathrm{stx}_{2 \mathrm{e}}$ in sows may provide mild protective immunity to pre-weaned pigs (Bertschinger 1999; Oanh et al. 2012).

Overall, the frequency of detecting the virulence genes was significantly high in the first week of life and from after the fourth week (post-weaning). These periods of increased detection of virulence genes roughly coincided with the period of the initiation of immunity (colostral) and waned maternal immunity (Oanh et al. 2012; Toledo et al. 2012). This study has indicated that virulence gene distribution in pigs from birth to the porker stage is diversely random and that EAST1 remains the most common gene during the growing period, which is in agreement with other studies (Choi et al. 2001; Osek 2003).

Antimicrobial usage in animals affects resistance patterns (Lanz et al. 2003; Mathew et al. 1999). Phenotypic AMR in E. coli was associated with pigs in both groups, evidenced even without antimicrobial usage in pigs, in which resistance levels were high. Because AM are used in commercial pig farms during farrowing as uterine suppositories or parenterally, vertical transmission of antimicrobial-resistant strains of microorganisms from the dam to piglets is highly likely with implications for genetic transfer of resistance genes among bacteria and consequent increased morbidity and mortality (Callens et al. 2015). Among the seven tested antimicrobial agents, E. coli isolates were most resistant to OT - this is consistent with findings of previous studies (Mathew et al. 1999; Van Den Bogaard, London \& Stobberingh 2000). Tetracyclines are widely used in the treatment of commonly observed pig diseases, and the presence of high concentrations of tetracycline in pig manure has been observed following the prophylactic use in sows ( $\mathrm{Li}$ et al. 2014). In addition to OT, the E. coli isolates were also resistant in high levels to AML and W similar to the findings in the Netherlands (Van Den Bogaard et al. 2000). In South Africa, the Fertilizers, Farm Feeds, Agricultural Remedies and Stock Remedies Act (Act 36 of 1947) subsists with laws that regulate antimicrobial administration in animals. Under this Act, tetracyclines, sulphonamides and penicillins are freely accessible over the counter and the records of use may not always be available (Henton et al. 2011). 


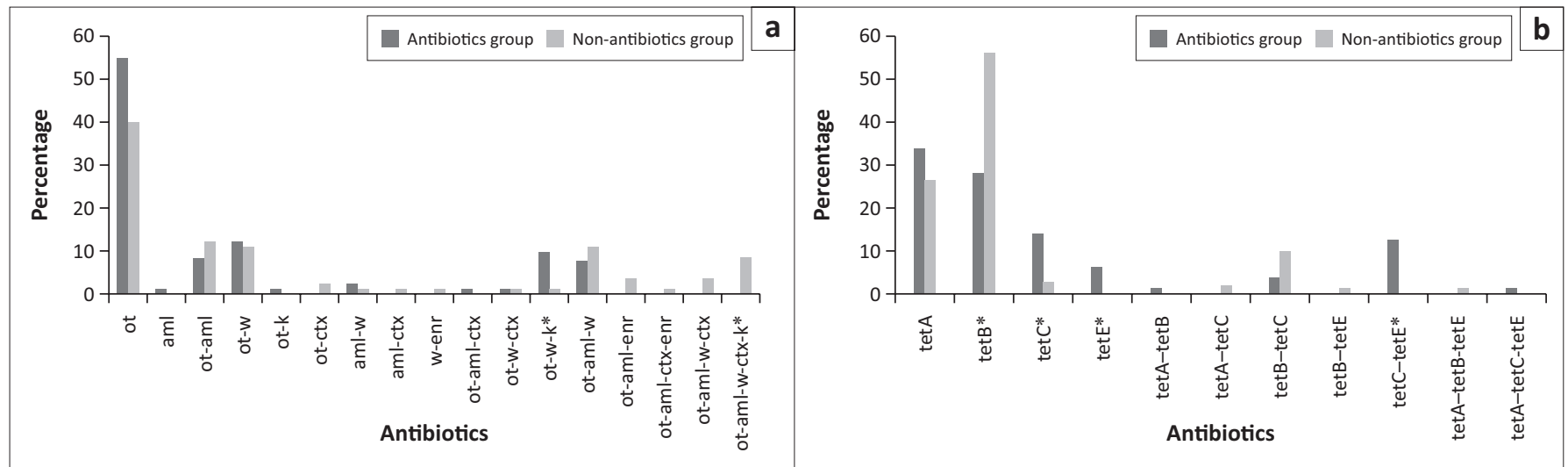

OT, oxytetracycline; AML, amoxicillin; W, trimethoprim; CTX, cefotaxime; K, kanamycin; ENR, enrofloxacin; FFC, florfenicol; tet, tetracyclines.

*, significant difference between antibiotic and non-antibiotic groups.

FIGURE 4: Frequency of (a) phenotypic combination of resistance among the seven antimicrobials tested and (b) genotypic combination of tet genes observed within each of the resistant Escherichia coli isolates for both groups.

Although resistance to OT and AML was significantly higher in the non-antimicrobial group, Callens et al. (2015) have earlier reached the same conclusion; however, it is at variance with the findings of another study (Österberg et al. 2016). High levels of tet (A, B, C) and E resistance genes were similarly observed in the study (Figure $4 a$ and b) and these confirmed the phenotypic patterns of OT resistance observed. The feeding of low levels of tetracycline, such as in growth promotion, may increase the chances of $E$. coli resistance genes development (Agga et al. 2014). Hence, the abundance of tet (A) and tet (B) genes in the isolates may result from the spread of $E$. coli clones carrying these genes as a result of selective pressures for the two genes. Whether this observation also has an environmental component to it is unknown. However, more piglets succumb to late-stage infections in this group and have to be humanely sacrificed. Considering that piglets live in an environment wherein the dam can pass the AMR gene in their faeces and milk, these factors may serve as predisposing conditions for environmentally acquired resistance organisms, with the possibility of multidrug resistance isolates. Resistance was least exhibited to ENR and CTX as detected phenotypically in our study. It should be understood that these substances are restricted for use in animals, and are only permitted under the stricter Act 101 that requires mandatory prescription by competent medical or veterinary personnel in South Africa (Henton et al. 2011).

Age-specific resistance patterns of isolates were observed, but were more pronounced within the second to fifth weeks of sampling. An association with increased usage of AM at this stage is feasible, as the increased risk of diarrhoea is observed during this period because of increased colonisation of the gut by pathogenic microorganisms. Piglets may also inadvertently ingest resistant strains of E. coli on the dams' teat during the process of suckling. The abundance of tet genes was observed in the period between birth and 35 days of age, possibly because of the frequent use of AM during this period to control and treat common diseases associated with piglets such as neonatal diarrhoea, post-weaning diarrhoea and oedema disease (Mathew et al. 1999). While a complex relationship exists between the concept of AMR and the development of virulence genes, yet this was beyond the scope of this study. It is, however, known that the transmission of antibiotic resistance and virulence has many parallel mechanisms (Schroeder, Brooks \& Brooks 2017).

\section{Conclusion}

This study has shown that virulence genes in pigs can develop and be observed at any stage during the growth phase with or without direct antimicrobial administration for prophylaxis or metaphylaxis. Perhaps a restriction of AM in growing meat-type pigs should be accompanied by a similar restriction in the associated production-type pigs. Phenotypic resistance to antimicrobial agents was high and randomly distributed throughout the growing period. Tetracycline (tet) genes are common in pigs because of high levels of use of tetracyclines.

\section{Acknowledgements}

The authors acknowledged the contribution of Prof. Don Cowan, A. Valverde and C.N. Kamutando and staff of the Bacteriology Laboratory of Agricultural Research CouncilOnderstepoort Veterinary Research for laboratory support during the course of this experiment, and also thank the management of the Lydia Albert's Pig Farm.

\section{Competing interests}

The authors have declared that no competing interests exist.

\section{Authors' contributions}

F.O.F. and R.H.A. conceived the project; F.O.F., O.G.F. and E.M. contributed to the material; R.H.A. and F.O.F. conducted the field work; R.H.A., O.A., F.O.F., E.M. and O.G.F. conducted the analysis; R.H.A., F.O.F. and E.M. wrote the initial draft; and all of the authors wrote and reviewed the final draft.

\section{Funding information}

This work was partially funded by the National Research Foundation Incentive Funding for Rated Researchers (IPRR) 
granted to F.O.F. (grant number: 103854). The University of Pretoria Postgraduate Bursary supported R.H.A. during the study.

\section{Data availability statement}

Data are available on the University of Pretoria website at the University of Pretoria electronic theses and dissertations (UPETD) website.

\section{Disclaimer}

The university or any organisation mentioned in this work is in no way liable for the content of this research. The authors are responsible for the contents and opinions expressed in this work.

\section{References}

Agga, G.E., Scott H.M., Amachawadi, R.G., Nagaraja, T.G., Vinasco, J., Bai J. et al., 2014 'Effects of chlortetracycline and copper supplementation on antimicrobial resistance of fecal Escherichia coli from weaned pigs', Preventive Veterinary Medicine 114(3-4), 231-246. https://doi.org/10.1016/j.prevetmed.2014.02.010

Barth, S., Schwanitz, A. \& Bauerfeind, R., 2011, 'Polymerase chain reaction-based method for the typing of F18 fimbriae and distribution of F18 fimbrial subtypes among porcine Shiga toxin-encoding Escherichia coli in Germany', Journal of Veterinary Diagnostic Investigation 23(3), 454-464. https://doi.org/10.1177/ 1040638711403417

Blanco, M., Lazo, L., Blanco, J.E., Dahbi, G., Mora, A., López, C. et al., 2006, 'Serotypes, virulence genes, and PFGE patterns of enteropathogenic Escherichia coli isolated
from Cuban pigs with diarrhea', International Microbiology 9(1), 53-60.

Bertschinger, H., 1999, 'Postweaning Escherichia coli diarrhea and edema disease', in B.E. Straw, S. D'Allaire, W.L. Mengeling, D.J. Taylor (eds.), Diseases of swine, in B.E. Straw, S. D'Allaire, W.L. Mengeling, D.J. Ta
pp. 441-454, lowa State University Press, Ames, IA.

Burow, E., Simoneit, C., Tenhagen, B.A. \& Käsbohrer, A., 2014, 'Oral antimicrobials increase antimicrobial resistance in porcine E. coli: A systematic review', Preventive Veterinary Medicine 113(4), 364-375. https://doi.org/10.1016/j.prevetmed. 2013.12.007

Callens, B., Faes, C., Maes, D., Catry, B., Boyen, F., Francoys, D. et al., 2015, 'Presence of antimicrobial resistance and antimicrobial use in sows are risk factors for antimicrobial resistance in their offspring', Microbial Drug Resistance 21(1) 50-58. https://doi.org/10.1089/mdr.2014.0037

Capita, R. \& Alonso-Calleja, C., 2013, 'Antibiotic-resistant bacteria: A challenge for the food industry', Critical Review in Food Science and Nutrition 53(1), 11-48. https:// doi.org/10.1080/10408398.2010.519837

Clinical Laboratory Standards Institute (CLSI), 2015, Performance standards for antimicrobial disk and dilution susceptibility tests for bacteria isolated from animals, 3rd edn., (VETO1S), viewed 09 February 2017, from https://clsi.org/ standards/products/veterinary-medicine/documents/vet01s/.

Chapman, T.A., Wu, X.Y., Barchia, I., Bettelheim, K.A., Driesen, S., Trott, D. et al., 2006, 'Comparison of virulence gene profiles of Escherichia coli strains isolated from healthy and diarrheic swine', Appllied and Environmental Microbiology 72(7), 4782-4795. https://doi.org/10.1128/AEM.02885-05

Cheng, D, Sun, H., Xu, J. \& Gao, S., 2005, 'Prevalence of fimbial colonization factors F18ab and F18ac in Escherichia coli isolates from weaned piglets with edema and/ or diarrhea in China', Veterinary Microbiology 110(1-2), 35-39. https://doi.org/ or diarrhea in China', Veterinary

Dean, A., Sullivan, K. \& Soe, M., 2015, OpenEpi: Open source epidemiologic statistics for public health, version, viewed 15 June 2017, from https://www.openepi.com/.

Dubreuil, J.D., Isaacson, R.E. \& Schifferli, D.M., 2016, 'Animal enterotoxigenic Escherichia coli', EcoSal Plus 7, 10. https://doi.org/10.1128/ecosalplus.ESP0006-2016

Choi, C., Cho, W., Chung, H., Jung, T., Kim, J. \& Chae, C., 2001, 'Prevalence of the enteroaggregative Escherichia coli heat-stable enterotoxin 1 (EAST1) gene in isolates in weaned pigs with diarrhea and/or edema disease', Veterinary Microbiology 81(1), 65-71. https://doi.org/10.1016/S0378-1135(01)00332-7

Enne, V.I., Cassar, C., Sprigings, K., Woodward, M.J. \& Bennett, P.M., 2008, 'A high prevalence of antimicrobial resistant Escherichia coli isolated from pigs and a low prevalence of antimicrobial resistant E. coli from cattle and sheep in Great Britain at slaughter', FEMS Microbiology Letters 278(2), 193-199. https://doi.org/10.1111/ j.1574-6968.2007.00991.x

Fasina, F.O.L., Bwala, D.G. \& Madoroba, E., 2015, 'Investigation of multidrug-resistant fatal colisepticaemia in weanling pigs', The Onderstepoort Journal of Veterinary Research 82(1), 986. https://doi.org/10.4102/ojvr.v82i1.986

Food and Drug Administration, 2012, 2012 Summary report on antimicrobials sold or distributed for use in food-producing animals, Department of Health and Human Services, viewed 15 November 2016, from https://www.federalregister.gov/ documents/2013/09/26/2013-23488/antimicrobial-animal-drug-sales-anddistribution-annual-summary-report-data-tables.
Gyles, C.L. \& Fairbrother, J.M., 2010, 'Escherichia coli', in C.L. Gyles, J.F. Prescott, J.G. Songer \& C.O. Thoen (eds.), Pathogenesis of bacterial infections in animals, pp. 267-308, Wiley-Blackwell, Hoboken, NJ.

Hawkins, P., Morton, D.B., Burman, O., Dennison, N., Honess, P., Jennings, M. et al., 2011, 'A guide to defining and implementing protocols for the welfare assessment of laboratory animals. Eleventh report of the BVAAWF/FRAME/RSPCA/UFAW joint working group on refinement', Laboratory Animals 45(1), 1-13. https://doi.org/ working group on refinem

Henton, M.M., Eagar, H.A., Swan, G.E. \& van Vuuren, M., 2011, 'Part VI. Antibiotic management and resistance in livestock production', South African Medical Journal 101(8), 583-586.

Ikwap, K., Larsson, J., Jacobson, M., Owiny, D.O., Nasinyama, G.W., Nabukenya, I. et al., 2016, 'Prevalence of adhesin and toxin genes in E. coli strains isolated from diarrheic and non-diarrheic pigs from smallholder herds in northern and eastern Uganda', BMC Microbiology 5/16(1), 178. https://doi.org/10.1186/s12866-016Uganda',

Kanengoni, A.T., Thomas, R., Gelaw, A.K. \& Madoroba, E., 2017, 'Epidemiology and characterization of Escherichia coli outbreak on a pig farm in South Africa', FEMS Microbiology Letters 364(3). https://doi.org/10.1093/femsle/fnx010

Lanz, R., Kuhnert, P. \& Boerlin, P., 2003, 'Antimicrobial resistance and resistance gene determinants in clinical Escherichia coli from different animal species in Switzerland', Veterinary Microbiology 91(1), 73-84. https://doi.org/10.1016/ S0378-1135(02)00263-8

Li, P., Wu, D., Liu, K., Suolang, S., He, T., Liu, X. et al., 2014, 'Investigation of antimicrobial resistance in Escherichia coli and enterococci isolated from Tibetan pigs', PLoS One 9(4), e95623. https://doi.org/10.1371/journal.pone.0095623

Luppi, A., Bonilauri, P., Dottori, M., Gherpelli, Y., Biasi, G., Merialdi, G. et al., 2015 'Antimicrobial resistance of F4+ Escherichia coli isolated from swine in Italy', Transboundary and Emerging Diseases 62(1), 67-71. https://doi.org/10.1111/ tbed.12081

Luppi, A., Gibellini, M., Gin, T., Vangroenweghe, F., Vandenbroucke, V., Bauerfeind, R. et al., 2016, 'Prevalence of virulence factors in enterotoxigenic Escherichia coli isolated from pigs with post-weaning diarrhoea in Europe', Porcine Health Management 2, 20. https://doi.org/10.1186/s40813-016-0039-9

Lutz, E.A., McCarty, M.J., Mollenkopf, D.F., Funk, J.A., Gebreyes, W.A. \& Wittum, T.E., 2011, 'Ceftiofur use in finishing swine barns and the recovery of fecal Escherichia coli or Salmonella spp. resistant to ceftriaxone', Foodborne Pathogens and Disease 8(11), 1229-1234. https://doi.org/10.1089/fpd.2011.0925

MacLeod, D., Gyles, C. \& Wilcock, B., 1991, 'Reproduction of edema disease of swine with purified Shiga-like toxin-II variant', Veterinary Pathology 28(1), 66-73. https://doi.org/10.1177/030098589102800109

Madzimure, J., Chimonyo, M., Zander, K.K. \& Dzama, K., 2012, 'Potential for using indigenous pigs in subsistence-oriented and market-oriented small-scale farming systems of Southern Africa', Tropical Animal Health and Production 45(1) 135-142. https://doi.org/10.1007/s11250-012-0184-3

Mathew, A.G., Saxton, A.M., Upchurch, W.G. \& Chattin, S.E., 1999, 'Multiple antibiotic resistance patterns of Escherichia coli isolates from swine farms', Applied and Environmental Microbiology 65(6), 2770-2772.

Meissner, H., Scholtz, M. \& Palmer, A., 2013, 'Sustainability of the South African livestock sector towards 2050 part 1: Worth and impact of the sector', South African Journal of Animal Science 43(3), 282-297. https://doi.org/10.4314/sajas. v43i3.5

Mohlatlole, R.P., Madoroba, E., Muchadeyi, F.C., Chimonyo, M., Kanengoni, A.T. \& Dzomba, E.F., 2013, 'Virulence profiles of enterotoxigenic, shiga toxin and enteroaggregative Escherichia coli in South African pigs', Tropical Animal Health and Production 45(6), 1399-1405. https://doi.org/10.1007/s11250-013-0377-4

Muniesa, M., Colomer-Lluch, M. \& Jofre, J., 2013, 'Could bacteriophages transfer antibiotic resistance genes from environmental bacteria to human-body associated bacterial populations?', Mobile Genetic Elements 3(4), e25847. https:// doi.org/10.4161/mge.25847

Ngeleka, M., Pritchard, J., Appleyard, G., Middleton, D.M. \& Fairbrother, J.M., 2003, Isolation and association of Escherichia coli AIDA-I/STb, rather than EAST1 pathotype, with diarrhea in piglets and antibiotic sensitivity of isolates', Journal of Veterinary Diagnostic Investigation 15(3), 242-252. https://doi.org/10.1177/ 104063870301500305

Oanh, T.K., Nguyen, V.K., De Greve, H. \& Goddeeris, B.M., 2012, 'Protection of piglets against Edema disease by maternal immunization with Stx2e toxoid', Infection and Immunity 80(1), 469-473. https://doi.org/10.1128/IAl.05539-11

Osek, J., 2003, 'Detection of the enteroaggregative Escherichia coli heat-stable enterotoxin 1 (EAST1) gene and its relationship with fimbrial and enterotoxin markers in E. coli isolates from pigs with diarrhoea', Veterinary Microbiology 91(1), 65-72. https://doi.org/10.1016/S0378-1135(02)00262-6

Österberg, J., Wingstrand, A., Nygaard, J.A., Kerouanton, A., Cibin, V., Barco, L. et al., 2016 'Antibiotic resistance in Escherichia coli from pigs in organic and conventional farming in four European countries', PLOS One 11(6), e0157049. https://doi. org/10.1371/journal.pone.0157049

Paton, J.C. \& Paton, A.W., 1998, 'Pathogenesis and diagnosis of Shiga toxin-producing Escherichia coli infections', Clinical Microbiology Reviews 11(3), 450-479. https:// doi.org/10.1128/CMR.11.3.450

Rice, L.B., 2009, 'The clinical consequences of antimicrobial resistance', Current Opinion in Microbiology 12(5), 476-481. https://doi.org/10.1016/j.mib.2009. 08.001

Roberts, M.C., 2011, 'Environmental macrolide-lincosamide-streptogramin and tetracycline resistant bacteria', Frontiers in Microbiology 2, 40. https://doi.org/ 10.3389/fmicb.2011.00040 
Schroeder, M., Brooks, B.D., Brooks, A.E., 2017, 'The complex relationship between virulence and antibiotic resistance', Genes (Basel) 8(1), 39. https://doi.org/ virulence and antibiotic

Sørum, H. \& Sunde, M., 2001, 'Resistance to antibiotics in the normal flora of animals', Veterinary Research 32(3-4), 227-241. https://doi.org/10.1051/vetres:2001121

Tadesse, D.A., 2012, 'Antimicrobial drug resistance in Escherichia coli from humans and food animals, United States, 1950-2002', Emerging Infectious Diseases 18(5), 741-749. https://doi.org/10.3201/eid1805.111153

Toledo, A., Gómez, D., Cruz, C., Carreón, R., López, J., Giono, S. et al., 2012, 'Prevalence of virulence genes in Escherichia coli strains isolated from piglets in the suckling and weaning period in Mexico', Journal of Medical Microbiology 61(Pt 1), 148-156. https://doi.org/10.1099/jmm.0.031302-0

Tripathi, V. \& Tripathi, P., 2017, 'Antibiotic resistance genes: An emerging environmental pollutant', in K.K. Kesari (ed.), Perspectives in environmental toxicology, pp. 183-201, Springer, Berlin. https://doi.org/10.1007/978-3-319-46248-6_9

Van Den Bogaard, A.E., London, N. \& Stobberingh, E.E., 2000, 'Antimicrobial resistance in pig faecal samples from the Netherlands (five abattoirs) and Sweden', The Journal of Antimicrobial Chemotherapy 45(5), 663-671. https://doi.org/10.1093/jac/45.5.663
Van den Bogaard, A.E. \& Stobberingh, E.E., 2000, 'Epidemiology of resistance to antibiotics: Links between animals and humans', International Journal of antibiotics: Links between animals and humans', International Journal of 00145-X

Vu Khac, H., Holoda, E., Pilipcinec, E., Blanco, M., Blanco, J.E., Mora, A. et al., 2006, 'Serotypes, virulence genes, and PFGE profiles of Escherichia coli isolated from pigs with postweaning diarrhoea in Slovakia', BMC Veterinary Research 2, 10 pigs with postweaning diarrhoea in Slovak
https://doi.org/10.1186/1746-6148-2-10

Wallace, J., Sanford, J., Smith, M. \& Spencer, K., 1990, 'The assessment and control of the severity of scientific procedures on laboratory animals', Laboratory Animals 24(2), 97-130. https://doi.org/10.1258/002367790780890185

World Health Organization, 2016, Critically important antimicrobials for human medicine, viewed 09 February 2017, from https://apps.who.int/iris/bitstream/ha ndle/10665/255027/9789241512220-eng.pdf;jsessionid=8830D9408E73E7DA20 B1D1E741121B40? sequence $=1$.

Zhao, L., Chen, X., Xu, X., Song, G. \& Liu, X., 2009, 'Analysis of the AIDA-I gene sequence and prevalence in Escherichia coli isolates from pigs with post-weaning diarrhoea and oedema disease', Veterinary Journal 180(1), 124-129. https://doi.org/ 10.1016/j.tvjl.2007.10.021 